\title{
Higgs boson production in association with a single top quark at the LHC
}

\author{
Oksana A. Koval ${ }^{1,2, \star}$, Igor R. Boyko ${ }^{1, \star \star}$, and Nazim Huseynov ${ }^{1, \star \star \star}$ \\ ${ }^{1}$ Dzhelepov Laboratory of Nuclear Problems, Joint Institute for Nuclear Research, Dubna, Moscow Region, \\ Russia \\ ${ }^{2}$ Obukhov Institute for Atmospheric Physics, Moscow, Russia
}

\begin{abstract}
Higgs boson production in association with a single top quark is the only process sensitive to the sign of the Top Yukawa coupling. We present a Monte-Carlo study of the $p p \rightarrow t H q b$ process and discuss the esperimental signatures that can help to discover it at the LHC. Two scenarios have been considered, the Standard Model case and the Inverted Top Coupling scenario.
\end{abstract}

\section{Introduction}

The discovery of the Higgs boson by ATLAS and CMS experiments in $2012[1,2]$ was the great success of the modern high-energy physics. After the Higgs boson discovery the Standard Model of electroweak interaction (SM) is complete. Currently the main goal of the LHC experiments is a search for New Physics (NP) phenomena beyond the SM. The effects beyond the Standard Model (BSM) can manifest themselves in two ways, either via a discovery of new particles or as small deviations of experimental observables from the SM predictions.

Precision measurements of the Higgs boson properties represent a promising field for the BSM searches. So far, all the measured properties of the Higgs boson are in agreement with the Standard Model predictions. The experimental observables studied at the LHC are the Higgs boson mass, spin and parity, decay branchings, kinematics of production, cross-sections of exclusive production modes.

Fig. 1 shows the energy dependence of cross-sections for different production modes of the Higgs boson. So far, 4 most abundant production modes have been observed at the LHC:

- gluon fusion $(87 \%, g g \rightarrow H)$

- vector boson fusion (7\%, $V V \rightarrow H$, where $V$ is $\mathrm{W}$ or $\mathrm{Z}$ boson)

- vector boson associated production (4\%, $p p \rightarrow H+V)$

- top pair associated production $(1 \%, p p \rightarrow t \bar{t} H)$.

A production of Higgs boson in association with a single top quark was not observed yet. For this production mode (which we call "signal" hereafter) the Standard Model predicts much smaller crosssection than for the Higgs boson production in association top quark pair. In this report we discuss the prospects of the signal discovery at the LHC.

\footnotetext{
^e-mail: kov.oksana20@gmail.com

$\star \star$ e-mail: Igor.Boiko@cern.ch

$\star \star \star$ e-mail: nazim.huseynov@cern.ch
} 


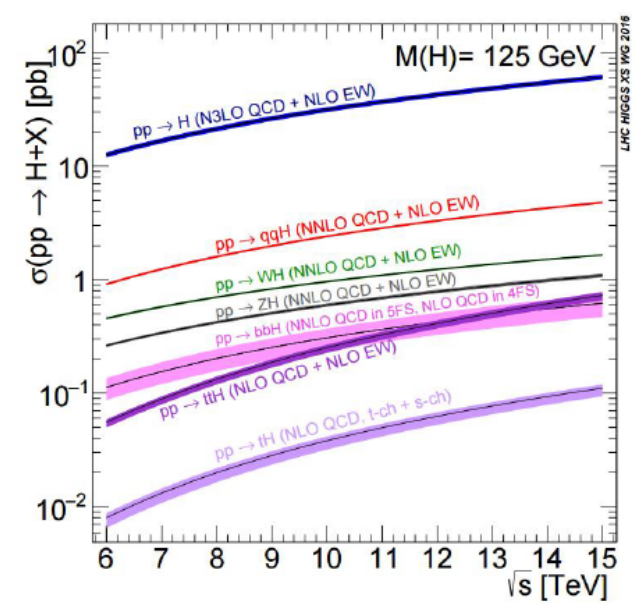

Figure 1. Energy dependence of the cross-section of the Higgs boson production in proton-proton collisions.

\section{The signal process}
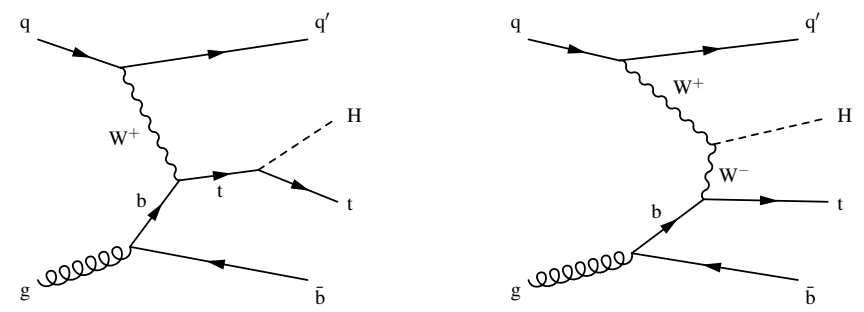

Figure 2. Feynman diagrams of the leading order signal processes.

The leading-order Feynman diagrams of the signal process are presented in Fig.2. One can see that the leading-order process is $p p \rightarrow t H q b$. The Higgs boson is produced in association with a single top quark, a b-quark from the gluon splitting and a light quark $(q)$ from the incoming beam.

Two diagrams contribute to the signal process, one is sensitive to the Higgs boson coupling to the W boson ( $W W H$ vertex), another to the Higgs boson coupling to the top quark ( $t \bar{t} H$ vertex). In the Standard Model there is a strong destructive interference between these two leading diagrams. As a result, the cross-section of the signal process is very small, approximately $71 \mathrm{fb}$ [3]. Such rare process can not be observed even after collection of the full LHC Run-2 statistics.

An observation of the signal would be a clear indication of a new physics beyond the Standard Model. The signal is sensitive not only to the couplings of the Higgs boson, but also to the relative phase between the $W W H$ and $t \bar{t} H$ vertices.

One of the key properties of the top quark is the top Yukawa coupling $Y_{t}$. The magnitude of the top Yukawa coupling has been already measured (indirectly) at LHC with $O(10 \%)$ precision. However, 
the $p p \rightarrow t H q b$ signal is the only process sensitive to the sign of $Y_{t}$, or, more generally, the phase difference between the $W W H$ and $t \bar{t} H$ vertices.

In particular, the signal cross-section is dramatically increased in the Inverted Top Coupling (ITC) scenario. In ITC all couplings are assumed to have the Standard Model magnitude, however the top Yukawa coupling is assumed to have an opposite sign with respect to SM. The interference between $W W H$ and $t \bar{t} H$ vertices becomes constructive rather than destructive, increasing the $t H q b$ production rate by an order of magnitude. The resultant signal cross-section (739 fb, [4]) makes it possible to observe a signal evidence with the statistics which is expected to be collected in 2016-2018 in the LHC Run-2.

\section{Generator-level study of the signal}

The signal properties have been studied using the MadGraph [5] event generator. The PDF set CT10 has been used. Events for both SM and ITC scenarios have been generated. The Higgs boson was forced to decay via the most abundant decay mode $H \rightarrow b \bar{b}$. The $\mathrm{W}$ boson from the top quark could decay either hadronically ( $\left.W \rightarrow q q^{\prime}\right)$ or leptonically $\left(W \rightarrow \ell v_{\ell}\right)$.

In the Standard Mode case the total production cross-section was calculated to be $32.1 \mathrm{fb}$ for the pp collisions at $\sqrt{s}=13 \mathrm{TeV}$. For the ITC scenario the cross-section is $413.5 \mathrm{fb}$. The calculated cross-sections take into account the branching fraction of the Higgs boson decay into the b-quark pair, $B F_{b \bar{b}} \sim 57 \%$.
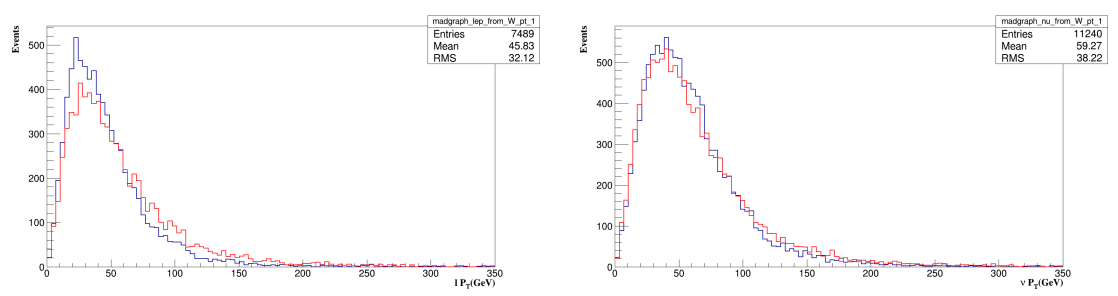

Figure 3. Transverse momenta of charged leptons (left) and neutrino (right) from the $\mathrm{W}$ decays. Blue histograms show the SM case, red histograms are for the ITC scenario.

Figure 3 shows the distributions of transverse momenta of leptons and neutrino from the semileptonic decays $t \rightarrow b W \rightarrow b \ell v_{\ell}$. In this and in the following figures the SM case is shown by a blue line, while the ITC scenario is shown by a red line. Histograms are normalized to an identical area. One can see that the distribution shapes are similar between SM and ITC cases and also between neutrino and charged leptons.

Figure 4 shows the distributions of the transverse momenta of b-quarks from Higgs boson and top quark decays. In both cases the $p_{T}$ is large enough for reliable jet reconstruction and b-tagging. As expected, the spectrum of b-quarks from top decays is somewhat harder than for the Higgs boson decays.

Figure 5 shows the distributions of total and transverse momenta of the light quarks $q$. The spectra are very hard since only a small fraction of the initial parton momentum is transferred in the t-channel $\mathrm{W}$ exchange. Fig.6 (left) shows the rapidity of the light quark $q$. The distribution is peaked toward 

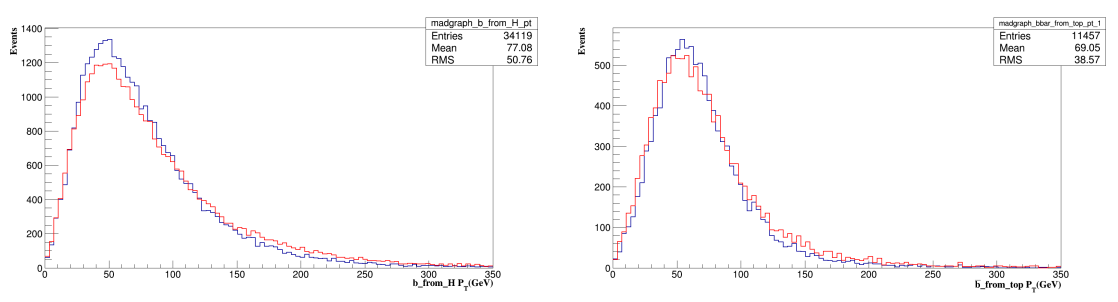

Figure 4. Transverse momenta of b-quarks from Higgs (left) and top quark (right) decays. Here and in the following figures blue histograms show the SM case, red histograms are for the ITC scenario.

the very high rapidity values. Finally, Fig.6 (right) shows the "rapidity gap", i.e. difference in rapidity between the light quark $q$ and the nearest b-quark from either Higgs or top decay. There is a significant difference between SM and ITC in the rapidity gap distribution. However, in both cases the value of the rapidity gap is significantly larger than zero.
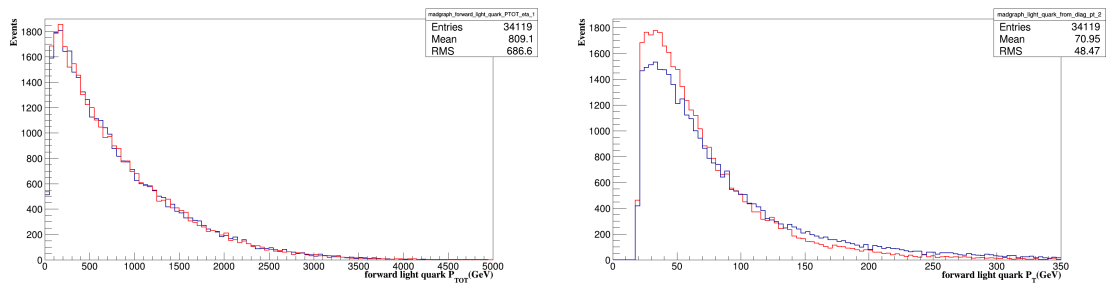

Figure 5. Total (left) and transverse (right) momenta of the light quark $q$.
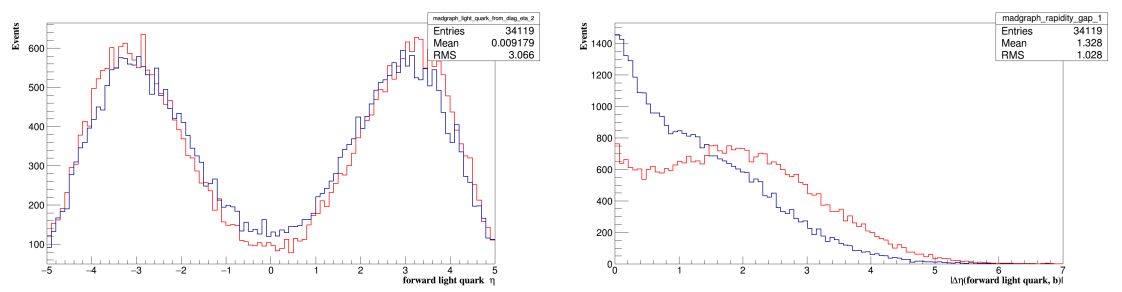

Figure 6. Rapidity of the light quark $q$ (left) and the rapidity gap (right). 


\section{Conclusions}

From the generator-level study presented above we conclude that the $p p \rightarrow t H q b$ signal can be searched using the decay chains $t \rightarrow b \ell v_{\ell}$ and $H \rightarrow b \bar{b}$. Events will be triggered by the energetic lepton with $p_{T}>30-40 \mathrm{GeV} / \mathrm{c}$. A similar cut can be applied to missing transverse momentum to account for the escaping neutrino.

A b-tagging can be used to select the b-jets from the top quark and Higgs boson decays. Finally, the very important feature of the signal is the presence of the very forward, very energetic jet which is characterized by a large rapidity gap with respect to the rest of the event.

\section{References}

[1] ATLAS Collaboration, Phys. Lett. B 716, 1 (2012)

[2] CMS Collaboration, Phys. Lett. B 716, 30 (2012)

[3] F. Demartin, F. Maltoni, K. Mawatari, and M. Zaro, Eur. Phys. J. C 75 no.6, 267 (2015)

[4] J. Alwall et al., JHEP 07, 079 (2014)

[5] J.Alwall et al., "The automated computation of tree-level and next-to-leading order differential cross sections, and their matching to parton shower simulations", arXiv:1405.0301 